\title{
Recent innovations in 3D-printed personalized medicines: an interview with Abdul Basit
}

\author{
Abdul Basit*,1 \\ ${ }^{1}$ UCL School of Pharmacy, University College London, 29-39 Brunswick Square, London WC 1N 1AX, UK \\ *Author for correspondence: a.basit@ucl.ac.uk
"'In 15 years' time, it is likely that 3D printers will be used throughout special manufacturing units and hospital pharmacies, enabling on-demand production of medicines for certain patient populations, diseases or medicines that require personalization,"

\begin{abstract}
Abdul Basit holds the position of Professor of Pharmaceutics at the UCL School of Pharmacy, University College London (UK). Basit is an internationally leading authority on oral drug delivery, digital health and innovative pharmaceutical technologies including 3D printing. Basit is a world authority on translational research; he has founded two spin-out companies (FabRx Ltd and Intract Pharma, both in London, UK) and has invented several drug products that have entered the clinic. More than a million patients to date have benefited from the treatments originating from his laboratory. Across his career, Basit has received prestigious awards from the American Association of Pharmaceutical Scientists, Academy of Pharmaceutical Sciences, GlaxoSmithKline and AstraZeneca and was listed among the world's most highly influential researchers in 2019.
\end{abstract}

First draft submitted: 11 May 2020; Accepted for publication: 13 May 2020; Published online:

1 June 2020

Keywords: drug delivery • multi-drug combinations • personalized medicines $\bullet$ pharmaceuticals $\bullet$ 3D printing

In this exclusive interview, Abdul Basit discusses the latest advances in 3D printing for drug development and his work within the field. This interview was conducted by Adam Price-Evans, the Managing Editor of the Journal of $3 D$ Printing in Medicine.

\section{Given your background in pharmaceutical science, how did you initially begin working with 3D printing technology?}

As a Pharmacist by training, ensuring patients receive the best therapies has always been at the forefront of my motivation for undertaking pharmaceutical research. I became interested in $3 \mathrm{D}$ printing because I saw that there was a real need for change in the way we treat patients. In the early 2010s, increasing evidence was showing the value in personalizing medicines for patients, however it was clear that the technologies used to produce medicines are not fit for purpose to meet this need. Indeed, for over 200 years, processes such as tableting and encapsulation have been used to make dosage forms, all of which are well suited to mass manufacture but not for the production of tailored, flexible dosage forms. We were determined to facilitate change and believed that 3D printing could make the entry of personalized medicines into pharmaceutical care a reality. As such, we pursued the development of 3D printing in pharmaceuticals and in 2013 purchased our very first 3D printer. The following year, we established FabRx Ltd, which is a spin-out biotech company from University College London focused on commercializing $3 \mathrm{D}$ printing technologies for pharmaceuticals and since then, we have developed a wide range of printing systems including the world's first pharmaceutical 3D printer for personalized medicines (M3DIMAKER ${ }^{T M}$ ). 
Your recent research is focused on the use of 3D printing technologies to develop personalized drug products. Why do you think personalized medicines are so important?

In recent years, there has been a considerable push to move away from treating patients via a 'one-size-fits-all' approach. It is becoming evident that one dose might not fit all; crucially, a report from NHS England (UK) showed that up to $70 \%$ of patients do not gain efficacy from the traditional mass manufacturing approaches [1]. It is now common knowledge that dosing can vary markedly based on a patient's individual characteristics, such as genetic profile, disease state, concurrent medicines and other factors (e.g., sex, weight and age). This understanding fashioned the field of personalized medicine, which involves tailoring therapies to a patient based on their individual characteristics, needs and preferences. Indeed, research has shown that by tailoring medicines (e.g., combining more than one drug into the same tablet or selecting appropriate dosages) enables improved medication adherence, improved safety and better therapeutic outcomes to be attained.

However, this poses a challenge if using conventional dosage forms (which are fixed strength), requiring tablets to be split or crushed and weighed to achieve the correct dose, increasing the risk of medication error and inconsistency. This is especially important for pediatric and geriatric patients, as well as for certain medicines that require exact tailoring (e.g., narrow therapeutic index drugs). A major benefit of $3 \mathrm{D}$ printing is that it enables $3 \mathrm{D}$ printed tablets to be designed, each with tailored aesthetics (shape, size, flavor and color), drug release, dose content and multiple drug combinations, enabling a truly personalized treatment approach for patients.

\section{Can you tell me more about your recent work in this area? What will be your focus going forward?}

The past 2 years have been very busy for us, and we have made significant progress in innovating the 3D printing field. In 2018, FabRx was awarded a grant totaling close to $£ 1$ million from Innovate UK (UK) to develop the world's first personalized medicine 3D printer. We have since launched the printing system (M3DIMAKER) which consists of a hardware system that is controlled by specialized software, allowing the selection of the required dose by the pharmacist according to the prescription given by the clinician. The system is fitted with advanced in-line quality control procedures, alongside camera monitoring of the printing process to track the progress and detect any faults during manufacture. A choice between three different printing nozzles allows the user to adapt the system to their manufacturing needs. The M3DIMAKER has a large array of applications within drug development (e.g., manufacture of small batches for preclinical and clinical studies), as well as in clinical practice (personalized medicines).

In 2019, we conducted a world first clinical study using 3D printed medicines in pediatrics. In the study, we integrated a 3D printer into the Pharmacy Department of the Clinic University Hospital in Santiago de Compostela (Spain) for the treatment of children (3-16 years old) with a severe metabolic disease called maple syrup urine disease. This innovation enabled us to produce $3 \mathrm{D}$ printed tablets or printlets ${ }^{\mathrm{TM}}$, in a variety of dosages, colors and flavors, which enabled a tighter control over target blood concentrations compared with the standard therapy (capsules). This study was a significant milestone in 3D printing history and demonstrates the true benefits of such technology in the pharmaceutical arena.

Going forward, we are focused on developing our current technologies and exploring new techniques for 3D printing. In 2019, we (FabRx) invented a revolutionary new 3D printing pharmaceutical system, known as direct powder extrusion, enabling the production of drug products in a single-step process directly from powdered materials. Critically, direct powder extrusion avoids the lengthy processes usually required to produce 3D printer filament feedstock using other methods, enabling flexible and tailored dosing with minimal development times.

\section{What do you think are the most promising recent innovations in this field? Which 3D printing technologies do you think could have a considerable impact on patient care?}

It is becoming apparent that a wide range of $3 \mathrm{D}$ printing technologies could have their place in improving patient care. While $3 \mathrm{D}$ printing is commonly thought as a single process, it is actually an umbrella term to describe a wide range of different methods. In the instance of personalized medicines, we believe the main technologies that will have benefit are extrusion-based (such as semi-solid extrusion, direct powder extrusion and fused deposition modeling), due to their compact, low cost and user-friendly nature. However, other printing types such as stereolithography and selective laser sintering also have their place, as they can produce unique drug products unattainable using conventional manufacturing technologies. As an example, using selective laser sintering we were able to produce fast-releasing orodispersible tablets that disintegrated in less than $4 \mathrm{~s}$. 
4D printing is emerging as a promising avenue of research in this field. How do you think this technology will affect drug delivery in the future?

$4 \mathrm{D}$ printing is a particularly exciting field of research that has entered pharmaceuticals in recent years. Built on the same underpinnings of $3 \mathrm{D}$ printing, the $4 \mathrm{D}$ printing method integrates a fourth-dimension, namely time. The printed products have the capability to change their configuration (e.g., change in shape, property or functionality) over time, often occurring in response to an external stimulus, such as heat, light, $\mathrm{pH}$, magnetic or electric forces or moisture. This provides a wide scope of possibility within medicine. Indeed, our group are focused on exploring the potential of $4 \mathrm{D}$ printing in the field of oral drug delivery by designing formulations that can respond to the changing environment of the GI tract (such as gastrointestinal fluid volume, composition and $\mathrm{pH}$ ). Such a technology is still in its infancy, but its potential to personalize a regime based on the internal environment is boundless.

\section{What are the major barriers facing the utilization of 3D printing technologies in drug development? What still needs to be done?}

As with any novel technology entering the pharmaceutical field, regulatory requirements are key considerations for the development of our $3 \mathrm{D}$ printing systems. A main challenge facing the use of $3 \mathrm{D}$ printing in pharmaceuticals involves ensuring the quality of the medicines produced. Previously, the major barrier to entry of this technology was the lack of 'pharmaceutical 3D printers', in other words, those that are licensed to produce medicines. In 2019, FabRx launched the M3DIMAKER pharmaceutical 3D printer, thus enabling the use of 3D printing in the pharmacy field for the first time. To ensure drug product quality, we have also developed and evaluated novel, nondestructive quality control methods which can be integrated into our $3 \mathrm{D}$ printing systems to enable a real-time check of medicine quality. To best guide us in this area, we are working closely with the regulatory bodies (including the UK's Medicines and Healthcare Products Regulatory Agency, the EMA and the US FDA) to enable us to best design $3 \mathrm{D}$ printers that will dispense medicines that are safe and effective for patients.

\section{Where do you see the field of 3D printing personalized drugs in 15 years' time?}

We envision a future where $3 \mathrm{D}$ printing plays a major part in pharmaceutical therapy. While printing technologies will not replace conventional mass manufacturing technologies, instead we think that it will be used to complement these processes to provide an alternative, flexible platform to enable true dose personalization at the click of a button. In 15 years' time, it is likely that 3D printers will be used throughout special manufacturing units and hospital pharmacies, enabling on-demand production of medicines for certain patient populations, diseases or medicines that require personalization, such as in the elderly, children and for those medicines with a narrow therapeutic index. We also foresee 3D printing entering the preclinical and clinical trials settings, due to the ability to rapidly produce drug products over a wide dose range by changing drug product dimensions or feedstock concentrations.

\section{Disclosure}

The opinions expressed in this interview are those of A Basit and do not necessarily reflect the views of Future Medicine Ltd.

Financial \& competing interests disclosure

A Basit is a founder of Intract Pharma and FabRx Ltd, whose research and products have been discussed within the present article. The interviewee has no other relevant affiliations or financial involvement with any organization or entity with a financial interest in or financial conflict with the subject matter or materials discussed in the manuscript apart from those disclosed.

\section{Reference}

1. NHS England. Improving outcomes through personalised medicine. (2016). www.england.nhs.uk/wp-content/uploads/2016/09/improving-outcomes-personalised-medicine.pdf 
